\title{
Mixed Color Sequential Technique for Reducing Color Breakup and Motion Blur Effects
}

\author{
Yi-Fu Chen, Che-Chin Chen, and Ke-Horng Chen
}

\begin{abstract}
This paper proposes a mixed color sequential (MCS) algorithm with high contrast enhancement technique in RGB light-emitting diode (LED) backlight display. Owing to synchronous control of liquid crystal display (LCD) and LED panels, high quality image with suppressed color breakup (CBU) and motion blur effects is achieved by our novel color sequential technique. Importantly, MCS algorithm is useful for color filter-less optical compensated bend (OCB) panel display for alleviating CBU and motion blur effects. Furthermore, high contrast image is also presented on LCD panel because of mixed red-green-blue (RGB) and cyan-magenta-yellow (CMY) backlights with optimum power consumption. In other words, MCS algorithm with high contrast enhancement technique can have the better performance compared with other field sequential color techniques. Experimental results demonstrate by an actual RGB backlight module for 32-in $1366 * 768 \mathrm{LCD}$ panel the improvement of $\mathrm{CBU}$ and motion blur effects.
\end{abstract}

Index Terms-Color breakup (CBU), color filter-less, color gamut, field sequential color, image quality, light-emitting diode (LED) backlight, liquid crystal display (LCD), motion blur, optical compensated bend (OCB).

\section{INTRODUCTION}

$\mathbf{T}$ HE choice of backlight can influence the performance of liquid crystal display (LCD) display. White light-emitting diode (LED) backlight has better color gamut than that of CCFL backlight. However, RGB LED backlight has the best color gamut, which is about $110 \%$ NTSC. The comparison of three popular backlight techniques is shown in Table I. Thus, in order to have compatible NTSC color gamut, the technique of RGB LED backlight for improving the display performance is a trend of today's LCD display market.

Owing to high power consumption in RGB LED backlight, color sequential techniques are proposed to reduce the power consumption without sacrificing color gamut. Besides, a CBUless pattern is needed to reduce the side effect of CBU [1]. In other words, we have to propose a novel color sequential technique to not only reduce the power consumption but also eliminate the $\mathrm{CBU}$.

Manuscript received January 24, 2007; revised February 27, 2007, and March 14, 2007. This work was supported by the National Science Council, Taiwan, R.O.C., under Grant NSC 95-2221-E-009-351.

The authors are with the Department of Electrical and Control Engineering, National Chiao Tung University, Hsinchu 300, Taiwan, R.O.C. (e-mail: khchen@cn.nctu.edu.tw).

Color versions of one or more figures are available online at http://ieexplore. ieee.org.

Digital Object Identifier 10.1109/JDT.2007.903160
TABLE I

COMPARISON BETWEEN THREE BACKLIGHT TECHNIQUES OF LCD

\begin{tabular}{|l|l|l|l|}
\hline & CCFL & White LED & RGB LED \\
\hline Saturation & Medium & Poor & Better \\
\hline Color Gamut & $60 \%$ NTSC & $70-80 \%$ NTSC & $110 \%$ NTSC \\
\hline $\begin{array}{l}\text { Lighten } \\
\text { efficiency }\end{array}$ & $60-801 \mathrm{~m} / \mathrm{W}$ & $15-201 \mathrm{~m} / \mathrm{W}$ & $15-201 \mathrm{~m} / \mathrm{W}$ \\
\hline Cost & Low & Medium & High \\
\hline Startup & Need warm-up & $\begin{array}{l}\text { Not need } \\
\text { warm-up }\end{array}$ & Not need warm-up \\
\hline Life & $\begin{array}{l}\text { Short } \\
\text { Better uniformity }\end{array}$ & $\begin{array}{l}\text { Long } \\
\text { Poor uniformity }\end{array}$ & $\begin{array}{l}\text { Long } \\
\text { Poor uniformity }\end{array}$ \\
\hline $\begin{array}{l}\text { Power } \\
\text { consumption }\end{array}$ & Medium & Low & High \\
\hline
\end{tabular}

As we know, it is very difficult to eliminate the motion blur in CCFL backlight technique because the flexibility of design CCFL backlight is low. However, for RGB backlight technique, the display method emulates impulse-type display compared to the hold-type display by CCFL backlight technique. Furthermore, it is very easy to insert black frames to eliminate motion blur effect for RGB backlight technique because of fast response of RGB LEDs.

In this paper, we propose a novel architecture to control the LCD and LED backlight, which is shown in Fig. 1. The architecture can simultaneously control the LCD and LED panels because $(\mathrm{R}, \mathrm{G}$, and $\mathrm{B})$ image data is not only sent to gate and source driver controllers (GCON and SCON) but also to backlight controller (BCON). According to the (R, G, and $\mathrm{B}$ ) image data sent to LCD controller, the strength of RGB backlight can synchronously respond to actual image data [2]-[4]. Color breakup and motion blur effects can be reduced by our mixed color sequential (MCS) algorithm because of synchronous backlight control. Our novel MCS algorithm with high contrast technique also enhances image quality. RGB backlight design architecture is shown in Fig. 2(a). Fig. 2(b) shows RGB backlight module for 32-in LCD panel with resolution $1366^{*} 768$. Fig. 2(c) and (d) shows the color sequential pattern and control circuits, respectively.

CBU and motion blur effects in conventional field sequential color display are explained in Section II; the MCS algorithm and high contrast enhancement technique is explained in Section III; Section IV presents experimental results and demonstrates the performance of MCS algorithm and high contrast enhancement technique. Furthermore, we also illustrate the high quality image can achieve optimum power consumption compared to conventional color sequential technique. Finally, we make a conclusion in Section V. 


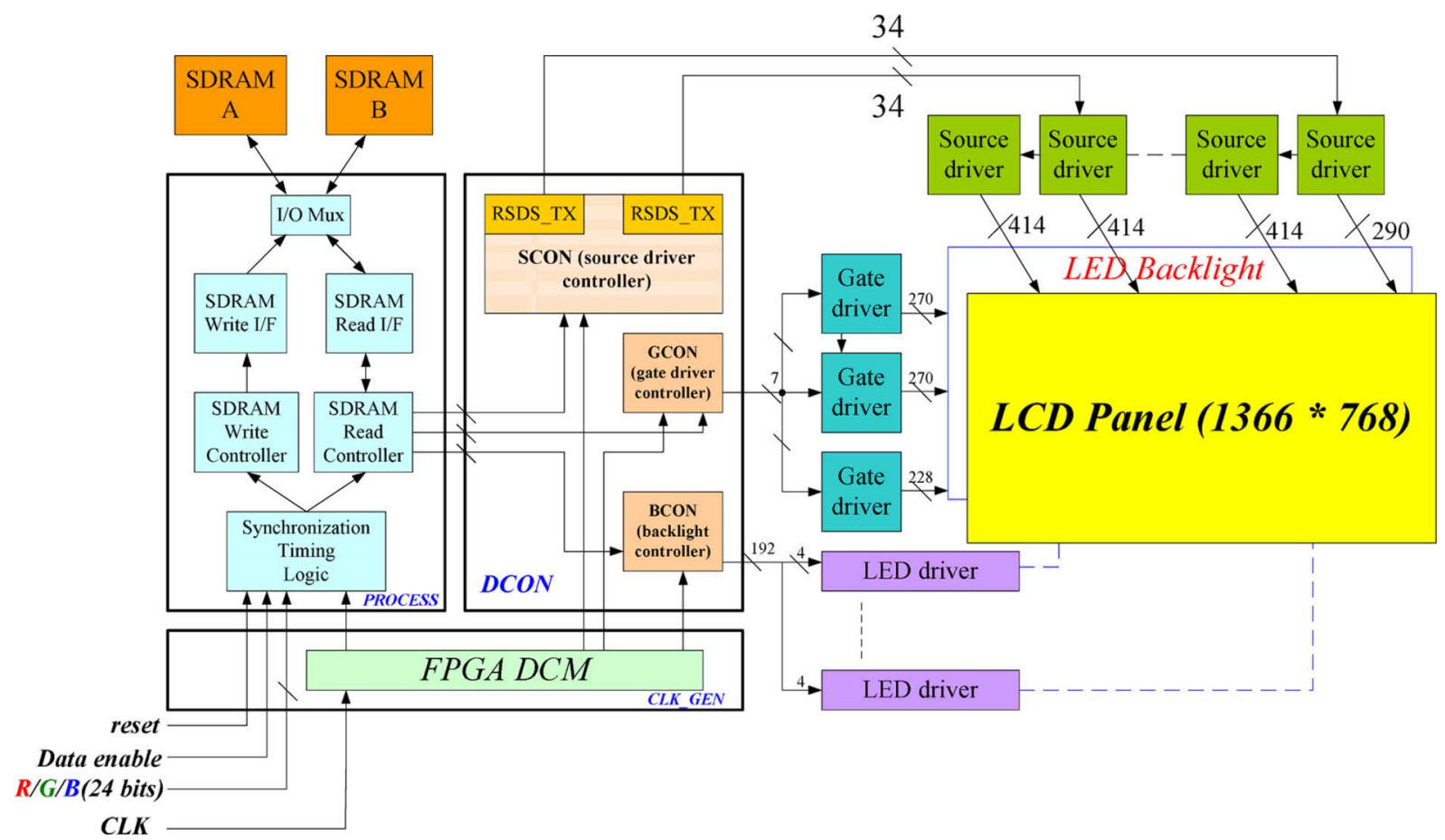

Fig. 1. System architecture for the proposed novel MCS algorithm.

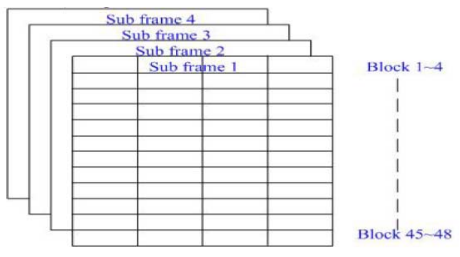

(a)

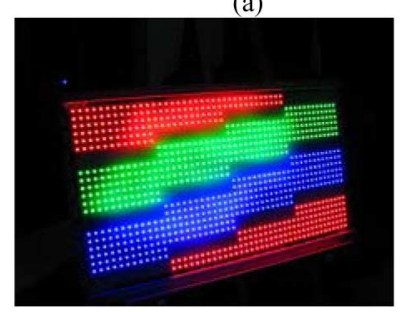

(c)

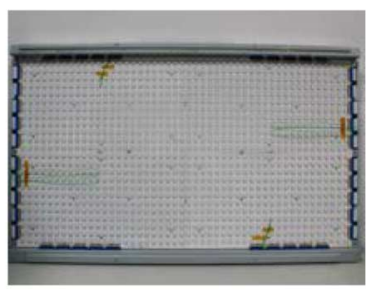

(b)

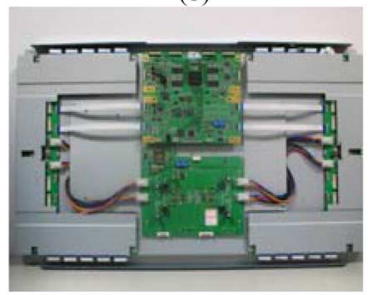

(d)
Fig. 2. RGB backlight design architecture (a) RGB backlight panel is composed of 48 blocks. (b) Photo of actual RGB backlight module for $1366 * 768$ 32-in LCD panel. (c) Color sequential pattern in our MCS algorithm. (d) Control circuits.

\section{CBU EFFects in Conventional Field SeQuential COLOR Display TeChNiQue AND MOTION BLUR EFFECTS IN LCD DISPLAY}

\section{A. CBU Effects}

Owing to the separation of color fields, an image frame with different color fields, which located on different area of human retina, cannot be viewed as a clear image with mixed color. The separation of different color fields is distinguished by human's vision system from a mixed color image frame. As we know, this phenomenon is CBU [5].

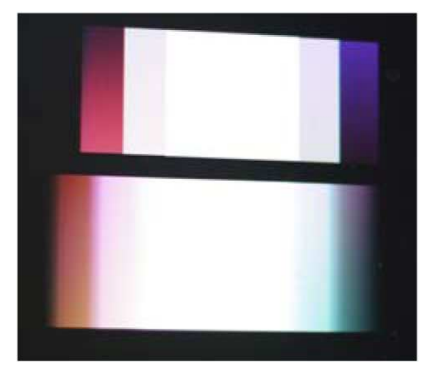

Fig. 3. Rainbow effect.

A moving object on FSC displays appears "rainbow effect" at the leading and tailing edges of the object. This effect named as "color breakup" (CBU) comes from sequential color techniques. Fig. 3 shows rainbow phenomenon caused by CBU effect. For a moving image, the sub-frames of moving objects are not plotted at the same spot for the smoothly moving retina. As a result, extra color fields are observed especially near the edges of the moving object. It is illustrated in Fig. 4 [6]. CBU is perceivable by vision systems where different sub-frame colors are observed separately. However, spatial and temporal variations can eliminate the CBU effect because smaller spatial division and higher temporal refresh rate can make human eyes fail to find rainbow effect. It means that high sub-frame rate and small spatial division improve the performance of color sequential technique.

\section{B. Motion Blur Effects}

The slow response time of the liquid crystal and hold-type driving technique in Fig. 5(a) are the main reasons that incur motion blur effects [7], [8]. The panel designers focus on fast response time of liquid crystal material. However, it needs much time to develop good performance of liquid crystal. Thus, we 


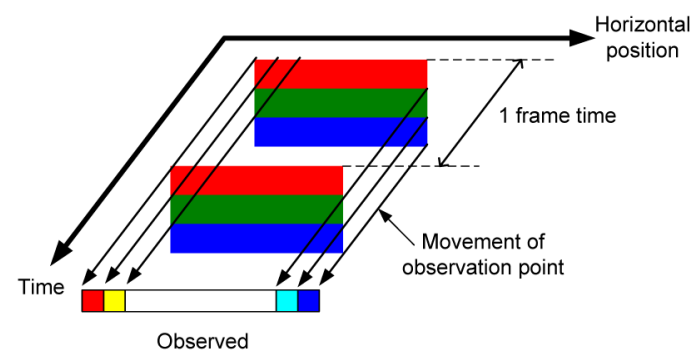

Fig. 4. Color breakup in a moving point.

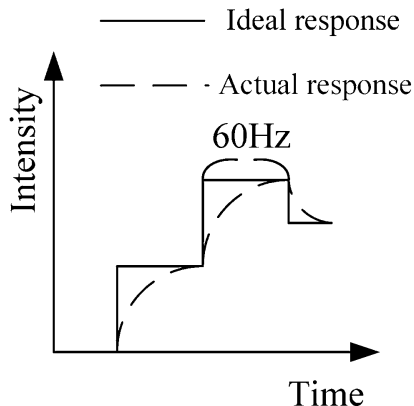

(a)

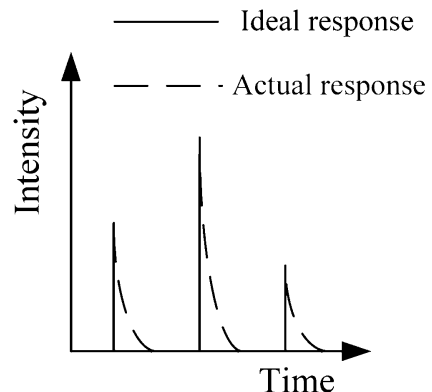

(b)
Fig. 5. (a) LCD Hold-type display. (b) CRT Impulse-type display.

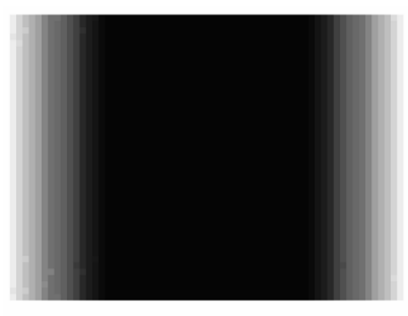

(a)

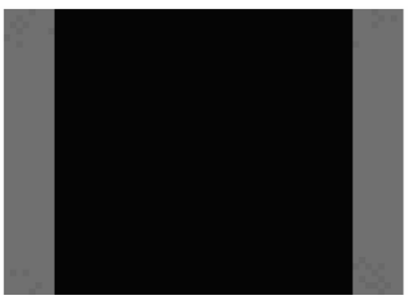

(b)
Fig. 6. (a) Movement of a square block by hold-type backlight technique. (b) Movement of a square block by impulse-type backlight technique.

can depend on the novel driving techniques to enhance image qualities with reduced motion blur effects.

For the LCD technique, image brightness is determined by the angle of liquid crystal, not by the backlight module with steady driving method. It means that the strength of backlight does not depend on the content of image. However, the display technique provided by cathode ray tube (CRT) is named as impulse-type technique shown in Fig. 5(b) because focused beam of electrons generated by CRT excite phosphor layer with red, green, and blue zones. Each pixel on the CRT screen is lightened owing to the excitation of focused beam of electrons. After excitation, the material can come back to its initial status. It is very difficult for the human's vision system to find out the process of excitation during a short period, as shown in Fig. 5(b) [9]. Contrarily, it is easy for the human vision system to find out the blur effect by the hold-type display technique. In Fig. 6(a), the movement of square block by hold-type backlight technique does not have motion blur effect. However, the movement of square block by

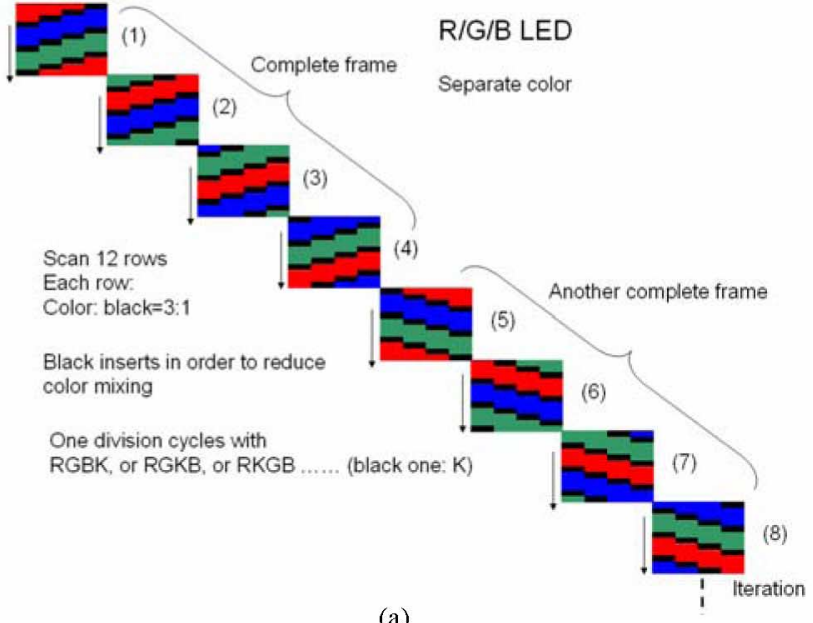

(a)

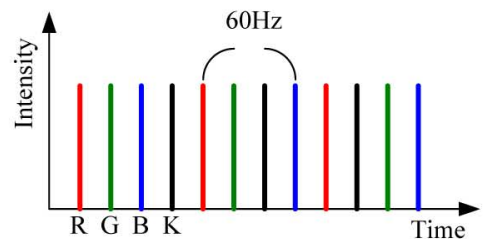

(b)

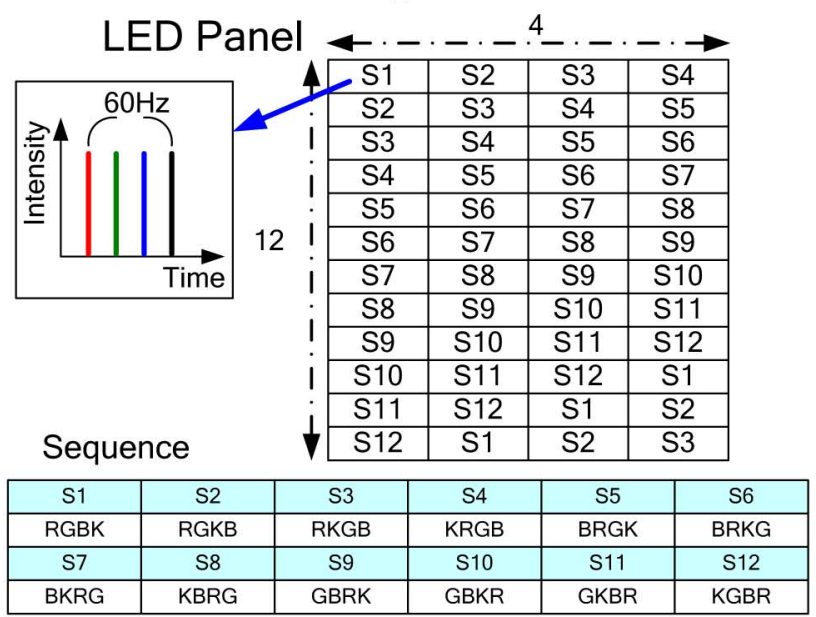

(c)

Fig. 7. (a) RGB color sequential technique. (b) The color sequence of the first block versus time. (c) Color sequence of each block.

impulse-type backlight technique has serious motion effect in Fig. 6(b).

\section{MCS TECHNIQUE FOR RGB BACKLIGHT SySTEM}

\section{A. RGB Color Sequential Technique}

In traditional LCD display, CCFL always emits white light and the light passes the color filter to become $\mathrm{R} / \mathrm{G} / \mathrm{B}$ three colors. The effect results in Motion Blur and the high power consumption. We propose a novel color sequential technique in Fig. 7(a), which uses pseudo-random sequences of color in the spatial and temporal domain on the LCD.

In the spatial, we divide a LED panel to many blocks and sequentially display different colors like red, green, blue, or black on each block. A LED frame is composed of four LED sub-frames. Therefore, the color sequence of each block in time 
domain is shown in Fig. 7(b) and (c). Besides, in order to further reduce CBU effect, we can add another type of four LED sub-frames as sub-frame 5 to 8 as shown in Fig. 7(a). When displaying moving images, the rainbow effect still happened. But with the new technique, the eyeballs of human find different colors having breakup at the same time. That is we cannot see through exact $\mathrm{CBU}$ when an object is moving. It means that this color sequential technique is successful to further suppress CBU effect in the visual system according to human's illusion.

As we know, if we want to implement this technique on color filter-less LCD panel, we must synchronously control the gray level of LCD panel. However, owing to slow response time of liquid crystal, we can only use four LED sub-frames in time domain. In other words, LED sub-frame rate is limited within $240 \mathrm{~Hz}$. Thus, spatial division for achieving small block size becomes another useful solution to alleviate CBU effects. Conversely, the LED sub-frame rate in LCD panel with color filter is not limited within $240 \mathrm{~Hz}$. Thus, we can increase the number of LED sub-frame from 4 sub-frames, to 16 sub-frames, or to 64 sub-frames because the control of LED backlight is not needed to be synchronized with that of LCD panel. It means that the probability of CBU found by human's eyes is small enough to achieve a high quality image. The iteration of color sequential technique is shown in Fig. 7(a). Higher sub-frame rate makes CBU effect lower than that of conventional color sequential techniques. Furthermore, the operation of MCS color sequential technique is emulated as impulse type in temporal domain. That is why the motion blur effect can be suppressed.

\section{B. CMY Color Sequential Technique}

In order to have high luminance without sacrificing color gamut, we propose Cyan-Magenta-Yellow (CMY) color sequential technique in LCD panel with color filter in Fig. 8(a) and (b) for high brightness [10]. This technique also uses pseudorandom sequence of color in the spatial and temporal domains on the LCD for eliminating CBU and motion blur effects.

CMY color sequential is generated by mixed RGB LED backlight. Compared to RGB color sequential technique, there are always two LEDs turned on among three RGB LEDs for every sub-frame. The luminance of CMY color sequential technique is raised and the effect of $\mathrm{CBU}$ is also reduced for displaying two of colors at the same time.

\section{High Contrast Algorithm}

In order to implement high contrast technique [11], [12] in our MCS algorithm in LCD panel with color filter, we raise LED sub-frame rate to display many complete sequences in one image frame period (1/60 second). Thus, the combination of CMY sequence, RGB sequence, and black insertion can constitute different brightness degree for high contrast display, which is shown in Fig. 9.
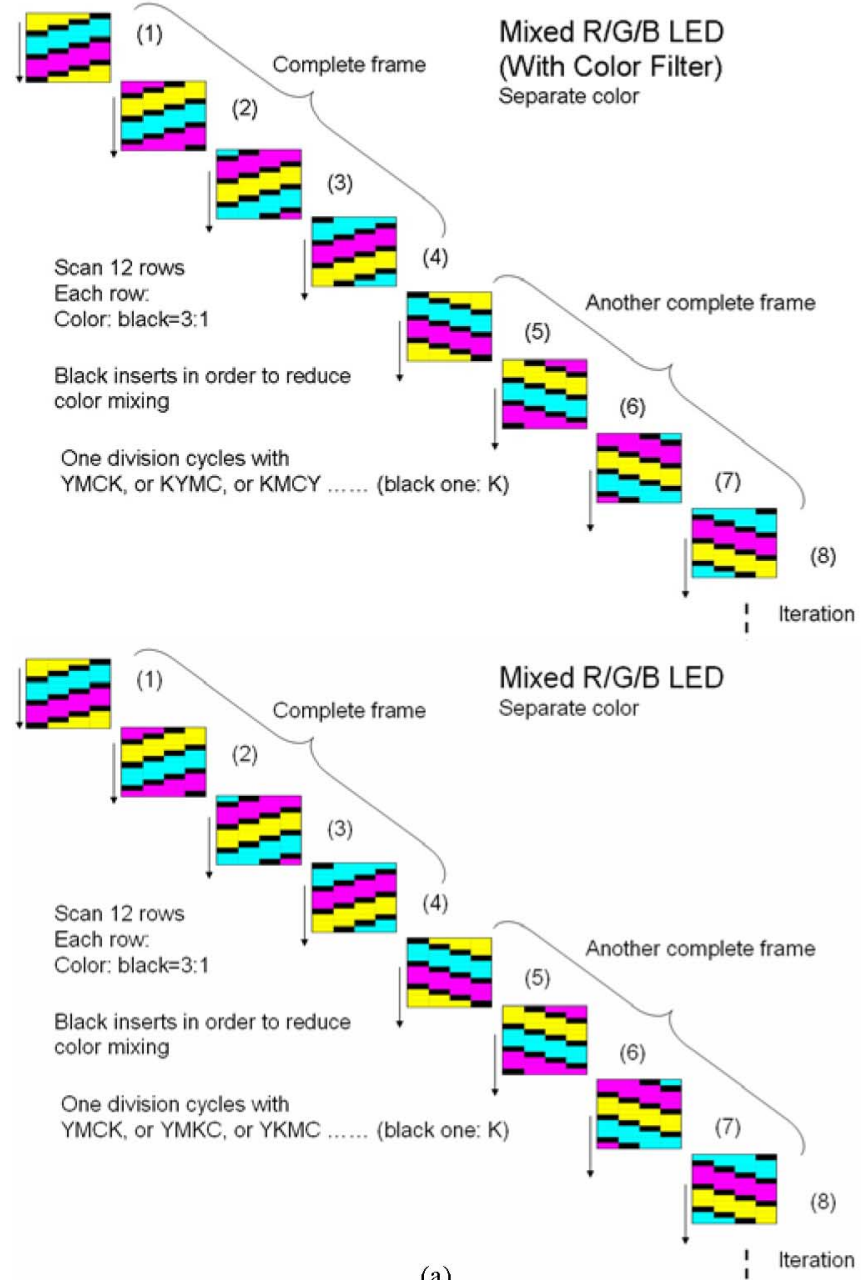

(a)

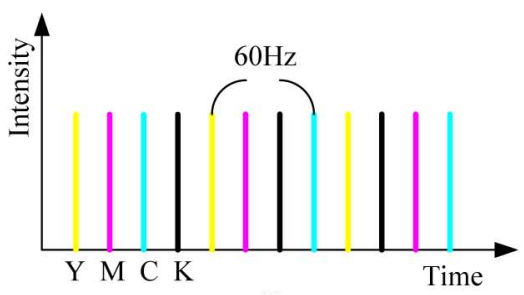

(b)

Fig. 8. (a) CMY color sequential technique. (b) Color sequence of the first block versus time.

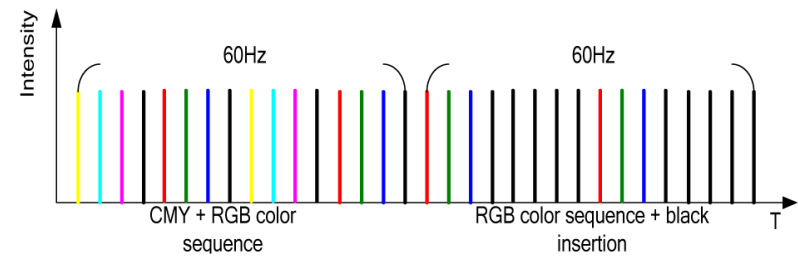

Fig. 9. Combination of CMY sequence, RGB sequence, and black insertion.

We combine two color sequential techniques to generate a low power high contrast backlight system. Generally speaking, the brightness of CMY color sequential technique is twice that of RGB color sequential technique. In other words, the combination RGB and CMY backlight can raise the image contrast. At first, we use a low resolution backlight panel to map to a high 


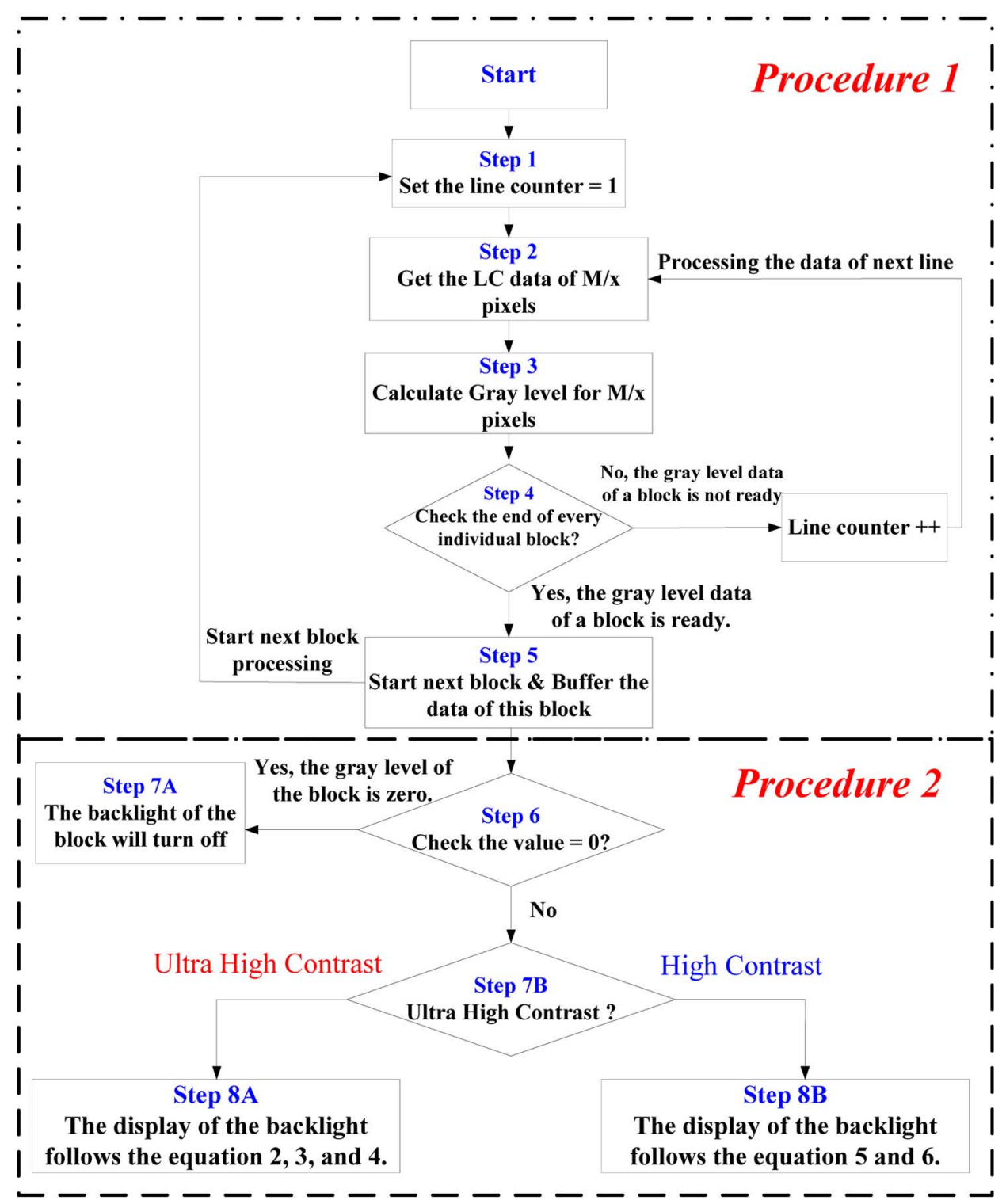

Fig. 10. Flowchart for the MCS algorithm with high contrast.

resolution LCD panel. It means that the backlight panel is designed as $x^{*} y$ regions to map to $\mathrm{M}^{*} N$ regions in LCD panel. Thus, each individual block contains $(M / x)^{*}(N / y)$ pixels. The decision of mixed backlight is the average LC gray level value of these pixels. The high contrast algorithm is implemented by the following two individual procedures. Two individual procedures can be executed at the same time because they are independent cases. The two procedures are shown in Fig. 10.

In procedure 1, we use (1) to calculate the average gray level value of block $N$ from steps 1 to 4 .

$$
V_{N}=\frac{\sum_{a=1}^{\frac{N}{y}} \frac{\sum_{a=1}^{\frac{M}{x}} P_{a}}{\frac{N}{x}}}{\frac{N}{y}} .
$$

$P_{a}$ stands for the gray level value of every pixel in block $N$. This procedure is finished when all gray level of every pixel in block $N$ is averaged. Then, $V_{N}$ stands for the average value of block $N$. If we finish the average of the individual block, we will decide the backlight patterns in procedure 2 .

In step 6, we make sure the average gray level is equal to zero or not. If the average gray level is zero, the backlight is turned off in step 7A. For nonzero average gray level value, we can decide the backlight patterns by step $8 \mathrm{~A}$ or $8 \mathrm{~B}$. If we turn on the ultra high contrast, the backlight patterns are decided by (2)-(4). Parameter $F$ is LED sub-frame rate and equal to four times a LED frame rate because a LED frame is composed of four sub-frames. Parameters $G$ and $Z$ are the maximum gray level of liquid crystal and the minimum backlight value of RGB, respectively. Equation (2) makes the backlight panel have higher contrast when low average gray level values are smaller or equal to $G / 2$. Based on minimum intensity of parameter $Z$, the ratio $V$ to $G$ determines the combination of backlight sequence, which is shown in right-side graph of Fig. 9. In other words, we use RGB patterns decided by (2) and black data insertion [13], [14] to display such a low gray level. However, we use RGB and CMY 
patterns to get a high image quality for higher gray level display, which is shown in left-hand side graph of Fig. 9. Equations (3) and (4) determine the mixed RGB and CMY patterns for the MCS technique

$$
\begin{aligned}
\operatorname{RGB}_{\text {pattern }}= & \operatorname{ROUND}\left(\left(\left(\frac{F}{4}-Z\right)\right)^{*} \frac{2 V}{G}+Z, 1\right) \\
& \text { for } V_{N} \leq \frac{G}{2} \\
\mathrm{RGB}_{\text {pattern }}= & \operatorname{ROUND}\left(\left(\frac{F^{*}}{4}\left(1-\frac{2 V-G}{G}\right)\right), 1\right) \\
& \text { for } V_{N}>\frac{G}{2} \\
\mathrm{CMY}_{\text {pattern }}= & \operatorname{ROUND}\left(\left(\frac{F^{*}}{4} \frac{2 V-G}{G}\right), 1\right) \\
& \text { for } V_{N}>\frac{G}{2}
\end{aligned}
$$

For high contrast mode, (5) and (6) decide the RGB and CMY patterns for MCS technique.

$$
\begin{aligned}
& \operatorname{RGB}_{\text {pattern }}=\operatorname{ROUND}\left(\left(\frac{F^{*}}{4}\left(1-\frac{V}{G}\right)\right), 1\right) \\
& \mathrm{CMY}_{\text {pattern }}=\operatorname{ROUND}\left(\left(\frac{F^{*}}{4} \frac{V}{G}\right), 1\right) .
\end{aligned}
$$

Due to mixed RGB and CMY patterns, we can get a high contrast display on LCD panel. Using optimum power consumption displays a high contrast image. Besides, we use black data insertion in ultra high contrast technique. It makes the contrast of image larger than that of conventional color sequential technique. The advantage of our MCS algorithm with high contrast enhancement technique is high contrast performance with optimum power consumption.

\section{MCS Technique on Optical Compensated Bend Panel}

Optical compensated bend panel utilizes special arrangement of liquid crystal molecules to implement angular compensation for good uniformity and wide view angle. Not similar to large switching liquid crystal on TN panel, OCB panel can have the same gray level with little switching liquid crystal under the same electric field. It means that OCB panel can have small response time.

Owing to four sub-frames provided by MCS technique, OCB panel without color filter needs to raise the sub-frame rate to about $240 \mathrm{~Hz}$. The absence of color filter on fast-response OCB panel means the synchronous data sequence of LCD and LED drivers is difficult for achieving correct color display. It means that MCS technique is suitable for color filter-less OCB panel by modifying the data sequences sent to LCD and LED drivers. For high contrast algorithm, we cannot raise the sub-frame rate because of limitation of OCB panel's response time. In other words, spatial enhancement dominates the performance of OCB panel. In the future, the decreasing response time of liquid crystal can also improve the image quality due to temporal enhancement. Mixed spatial and temporal technique can have the best display performance.

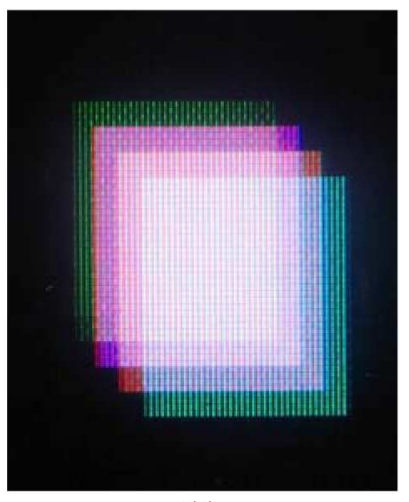

(a)

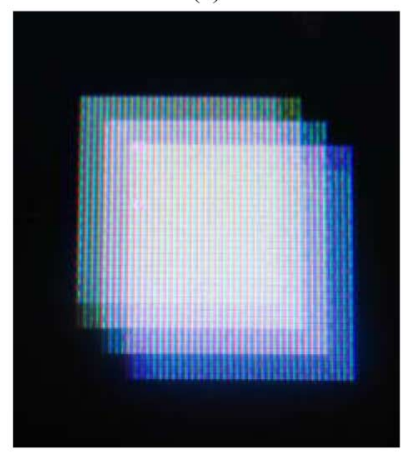

(b)

Fig. 11. (a) Color breakup owing to conventional color sequential technique. (b) Improved CBU by RGB color sequential technique. (The overlapped white square objects are due to the speed of camera shutter is larger than that of original moving object.).

\section{EXPERIMENTAL RESULTS}

\section{A. RGB Color Sequential Technique}

It is obvious that RGB color sequential technique can improve CBU effect compared to conventional color sequential technique because of spatial and temporal display technique. Experimental environment is: The rate of shutter is $1 / 25$ second and the size of white square block is 36 pixels *38 pixels. The moving speed of white square block from left-top to right-bottom is $240 *(\sqrt{ } 2)$ pixel/s. In Fig. 11(a), we can observe separate RGB square objects on LCD panel without color filter. It means that conventional color sequential technique suffers serious CBU effect. The reason of serious CBU effect is that it is very easy to find out CBU effect by human's eyes for separate RGB frames. It is the disadvantage of only temporal technique for solving CBU effect. In other words, we have to adapt spatial technique to color sequential technique for improving the $\mathrm{CBU}$ effect. Owing to mixed temporal and spatial color sequential technique, the illusion of mixed color sequence reduces the CBU generated by color sequential technique. The improvement is shown in Fig. 11(b). Generally speaking, mixed CBU in every small area dexterously make human's eyes hard to distinguish clear color from mixed CBU.

In order to estimate the effects of motion blur, a white square object with black background moves from left-top to right-bottom on different panels, which are CRT panel, LCD panel with CCFL backlight module, and OCB LCD panel (color filterless panel) with RGB backlight module. According 


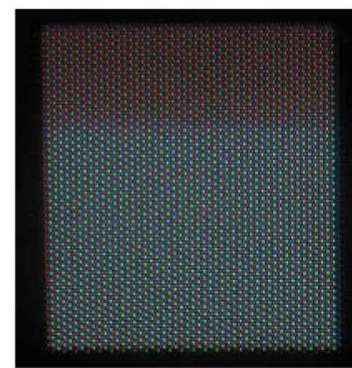

(a)

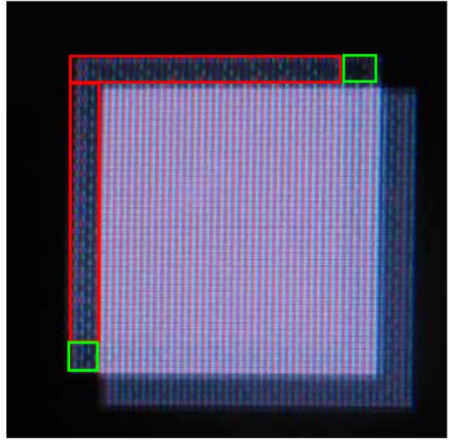

(b)

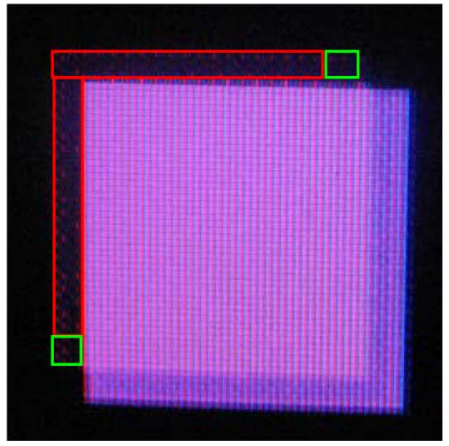

(c)

Fig. 12. (a) Moving object on conventional CRT display. (b) Motion blur owing to moving object on liquid crystal display by CCFL hold-type backlight module. (c) Motion blur owing to moving object on liquid crystal display by RGB impulse-type backlight module.

to the pictures taken by digital camera, we can observe that there is no motion effect on CRT panel because of impulse-type display technique, which is shown in Fig. 12(a).

Owing to the different brightness between in red and green windows, we can observe obvious blur images in red window in Fig. 12(b), which is estimated on LCD panel with CCFL backlight module. After the receiver of human's eyes, it is easy to find out blur effect at the edge of white square object [15]. However, the blur effect can be alleviated by RGB color sequential technique implemented on OCB LCD panel (color filterless panel) with RGB backlight module because of impulse-type display. The brightness of blur images in red window can be ignored by RGB color sequential technique in Fig. 12(c), which shows the alleviation of blur effect. The blur effect is shrunk from a red window in Fig. 12(b) to few blur pixels in red window in Fig. 12(c).

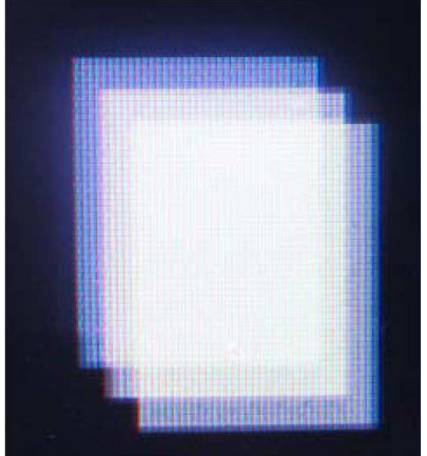

Fig. 13. Color breakup owing to CMY color sequential technique.

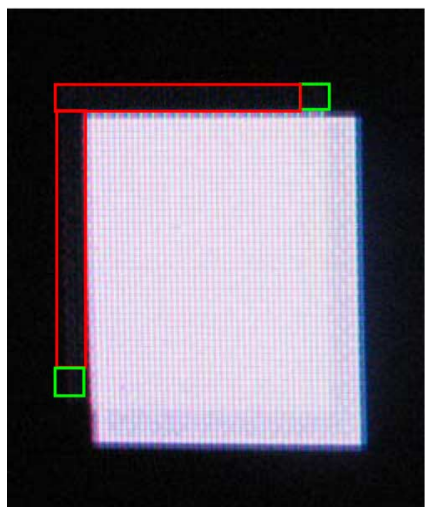

Fig. 14. Reduced motion blur effect in CMY color sequential technique compared to RGB color sequential technique.

\section{B. CMY Color Sequential Technique}

Owing to the absence of color is only one color in RGB backlight module by replacing RGB color sequential technique by CMY color sequential technique, the CBU effect of CMY color sequential technique is less than that of RGB color sequential technique. The comparison between Fig. 13 and Fig. 11(b) can illustrate the improvement benefited from CMY color sequential technique. Although, the improvement of motion blur by CMY color sequential technique is the same as that by RGB color sequential technique. It is obvious that the image quality is improved by CMY color sequential technique because of reduced blur effect, which is shown in Fig. 14, which implemented on OCB LCD panel (color filterless panel). As we expect, the blur effect in red window is better than that generated by RGB color sequential technique. However, the color gamut is lightly reduced because of OCB LCD panel.

\section{High Contrast Algorithm}

The experimental color gamut of our RGB backlight is about $102.5 \%$ NTSC. The comparison of color gamut is shown in Fig. 15. As we expect, it is larger than that of CCFL backlight technique.

A photo in Fig. 16, which contains a sun with high brightness, is used to demonstrate the performance of MCS algorithm. It seems that the image with uniform RGB backlight does not have high contrast only by gray level of LC. Conversely, the 


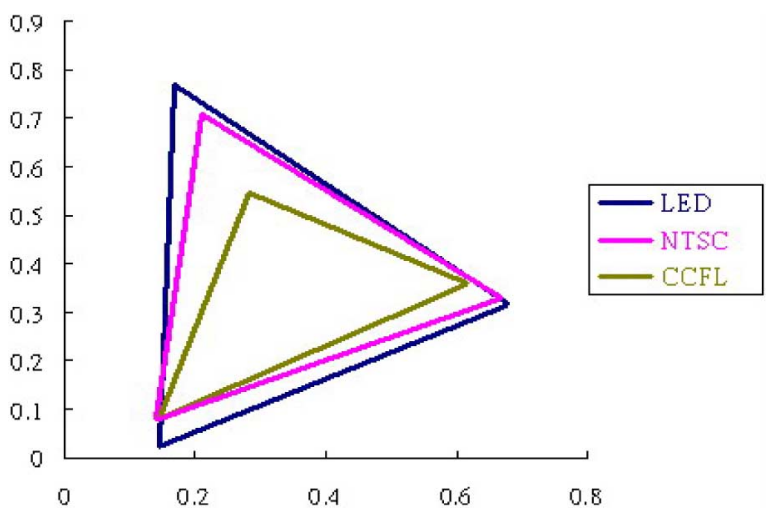

Fig. 15. CIE color chart.

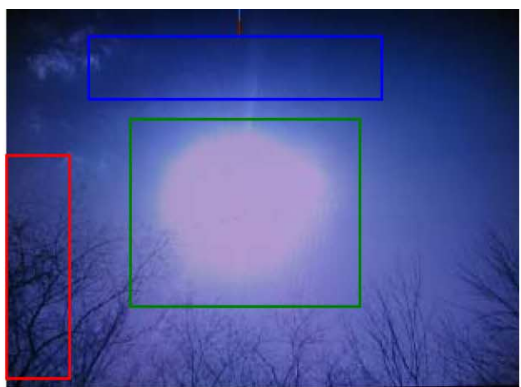

(a)

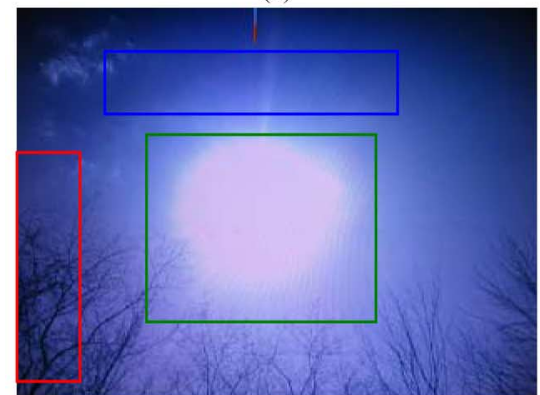

(b)

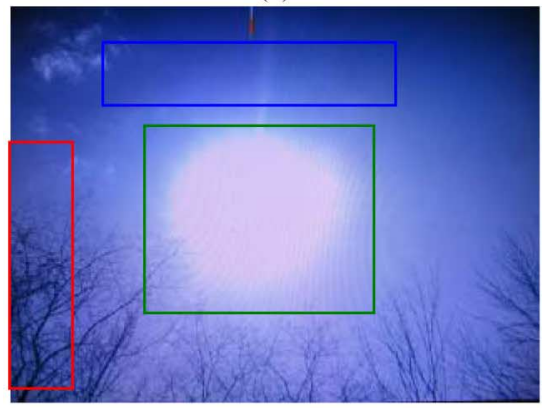

(c)

Fig. 16. (a) Only RGB color sequential technique. (b) Combination RGB and CMY color sequential techniques. (c) Only CMY color sequential technique.

image with uniform and high power CMY backlight shows high brightness for whole image. However, the image contrast is not improved by uniform CMY backlight because of whole image with high brightness.

However, with our proposed high contrast technique, the sun has the highest brightness can be enhanced by CMY color sequential technique. The rest of the image is also enhanced by the calculation of gray level in each individual block. In other

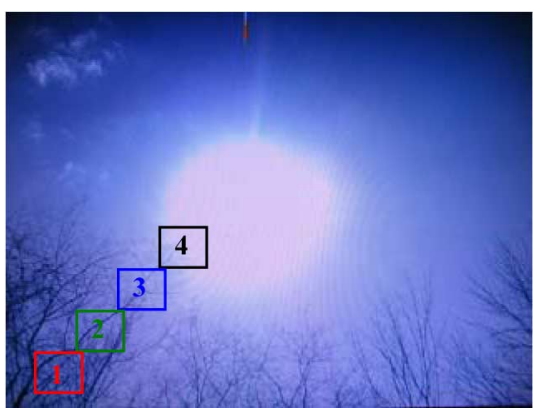

Fig. 17. Original image displayed by CCFL backlight.

TABLE II

COMPARISON OF POWER CONSUMPTION OF THREE DifFERENT BACKLIGHT TECHNIQUES

\begin{tabular}{|l|l|l|l|}
\hline $\begin{array}{r}\text { Mode } \\
\text { Block }\end{array}$ & $\begin{array}{l}\text { RGB } \\
\text { backlight }\end{array}$ & $\begin{array}{l}\text { CMY } \\
\text { backlight }\end{array}$ & $\begin{array}{l}\text { MCS } \\
\text { Algorithm }\end{array}$ \\
\hline Block1 & $2.484 \mathrm{w}$ & $4.968 \mathrm{w}$ & $3.260 \mathrm{w}$ \\
\hline Block2 & $2.484 \mathrm{w}$ & $4.968 \mathrm{w}$ & $3.726 \mathrm{w}$ \\
\hline Block3 & $2.484 \mathrm{w}$ & $4.968 \mathrm{w}$ & $4.502 \mathrm{w}$ \\
\hline Block4 & $2.484 \mathrm{w}$ & $4.968 \mathrm{w}$ & $4.968 \mathrm{w}$ \\
\hline Average & $2.484 \mathrm{w}$ & $4.968 \mathrm{w}$ & $4.114 \mathrm{w}$ \\
\hline
\end{tabular}

words, a high contrast enhancement technique can save much power consumption compared to the white LED backlight technique. Importantly, we use optimum power consumption to enhance the contrast of the image. High quality display can be achieved by our MCS algorithm with high contrast enhancement technique.

We point out the difference between three color sequential techniques by three square regions from Fig. 16(a)-(c). Owing the highest brightness in Fig. 16(c), the details of gray level in red square region cannot be obvious for our eyes. However, for green square region, the sun can be expressed perfectly by Fig. 16(b) and (c). Fig. 16(a) cannot present a bright sun. Furthermore, owing to incremental brightness expressed by Fig. 16(b), the details of blue square region can be displayed according to actual image gray level. It is the advantage of our MCS algorithm with high contrast enhancement technique.

Fig. 17 and Table II show the power consumption of four distinct blocks. It is obvious that high contrast enhancement technique is achieved by little increase in power compared to RGB color sequential technique.

\section{CONCLUSION}

The MCS algorithm with high contrast enhancement technique in RGB LED backlight display achieves high quality image with suppressed CBU and motion blur effects. Furthermore, high contrast image with optimum power consumption is also presented in LCD panel because of mixed RGB and CMY backlights. In other words, MCS algorithm with high contrast enhancement technique can have the better performance compared with other field sequential color techniques. Furthermore, the MCS technique is still developed for color filter-less OCB panel. The optimum solution of mixed spatial and temporal technique will be achieved by our MCS technique. 


\section{ACKNOWLEDGMENT}

The authors wish to thank Chunghwa Picture Tubes, Ltd., for their help.

\section{REFERENCES}

[1] K. Käläntär, T. Kishimoto, K. Sekiya, T. Miyashita, and T. Uchida, "Spatio-temporal scanning backlight for color-field sequential optically compensated bend liquid-crystal display," SID'05 Dig. $36.3 \mathrm{~L}$, pp. 1316-1319.

[2] T. Shirai, S. Shimizukawa, T. .Shiga, S. Mikoshiba, and K. Kalantar, "RGB-LED backlights for LCD-TVs with 0D, 1D, and 2D adaptive dimming," SID'06 Dig. 44.4, pp. 1520-1523.

[3] J. B. Eichenlaub, "Field sequential color illumination system for liquid crystal display," U.S. Patent 5 428 366, Jun. 27, 1995.

[4] K. Miyazawa, "Panel type color display device and system for processing image information," U.S. Patent 6078 304, Jun. 20, 2000.

[5] A. Yohso and K. Ukai, "How color break-up occurs in the human visual system: Mechanism of color break-up phenomenon," SID'06 Dig. 25.2, pp. 1223-1228.

[6] R. Van Dijk and J. A. Shimizu, "A system and method for motion compensation of image planes in color sequential displays," U.S. Patent 6831948, Dec. 14, 2004

[7] S. Hong, B. H. Shin, T.-S. Kim, B. Berkeley, and S. S. Kim, "Advanced method for motion blur reduction in LCDs," SID'05 Dig. P-51, pp. 466-469.

[8] K. H. Shin, J. Y. Ahn, K. D. Kim, H. H. Shin, and I. J. Chung, "Acceptable motion blur levels of a LCD TV based on human visual system," SID' 06 Dig. P-25, pp. 287-290.

[9] T. Kurita, "Moving picture quality improvement for hold-type AM-LCDs," SID'01 Dig., pp. 986-989.

[10] T. Shiga, S. Kuwahara, N. Takeo, and S. Mikoshiba, "Adaptive dimming technique with optically isolated lamp groups," SID'05 Dig. 18.1, pp. 992-995.

[11] J. Bergquist and C. Wennstam, SID’06 Dig. 49.2, pp. 1594-1597.

[12] J. H. Stessen and J. G. R. van Mourik, "Algorithm for contrast reserve, backlight dimming, and backlight boosting on LCD," SID'06 Dig. 26.4, pp. 1249-1252.

[13] J. Hirakata, A. Shingai, Y. Tanaka, K. Ono, and T. Furuhashi, "SuperTFT-LCD for moving picture images with the blink backlight system," SID'01 Dig. 35.2, pp. 990-993.

[14] H. Pan, X. Feng, and S. Daly, "Quantitative analysis of LCD motion blur and performance of existing approaches," SID'05 Dig. 51.4, pp. 1590-1593.
[15] K. Sekiya and N. Nakamura, "Eye-trace integration effect on the perception of moving pictures and a new possibility for reducing blur on hold-type displays," SID'02 Dig., pp. 930-933.

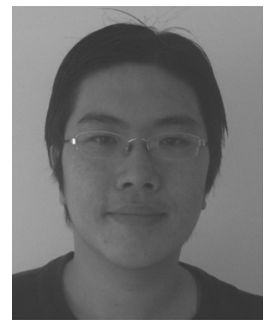

Yi-Fu Chen received the B.Sc. degree in electrical engineering from the University of Feng-Chia, Taiwan. He is currently a MSc student in College of Electrical and Computer Engineering, National Chiao Tung University and his research interests include LCD display algorithm, SoCs and microprocessor.

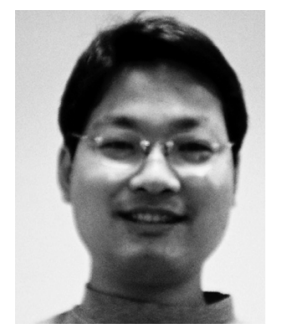

Che-Chin Chen received the B.S. degree in electronic engineering from the University of Chung-Yuan, Taiwan, in 1999 and the MS degree in College of Electrical and Computer Engineering, National Chiao Tung University, Taiwan in 2007. His current topic includes flat panel display algorithm and design. Previous topic includes development of active matrix organic light emitting diode and driving circuit design.

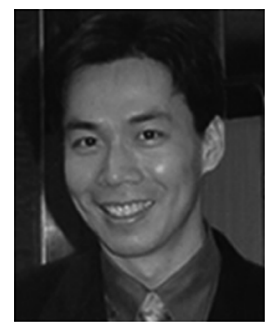

Ke-Horng Chen was born in Taipei, Taiwan, R.O.C., in 1971. He received B.S., M.S., and Ph.D. degrees in electrical engineering from National Taiwan University (NTU), Taipei, in 1994, 1996 and 2003, respectively. From 1998 to 2000, he was an application engineer in Avanti, Ltd. During 2000-2003, he was a project manager in ACARD, Ltd., where he worked on the developing of the power management IC. Since 2004, he has been with National ChiaoTung University, Taiwan, where he is now an Assistant Professor. His current research interests include power management IC designs, mixed-signal circuit designs, LCD display algorithm, and low-voltage circuit designs. 